\title{
Self-Assembly of Bicomponent Molecular Monolayers: Adsorption Height Changes and Their Consequences
}

\author{
E. Goiri, ${ }^{1}$ M. Matena, ${ }^{1}$ A. El-Sayed, ${ }^{2}$ J. Lobo-Checa, ${ }^{3}$ P. Borghetti, ${ }^{1,3}$ C. Rogero, ${ }^{1,3}$ B. Detlefs,,${ }^{4,}$ \\ J. Duvernay, ${ }^{4}$ J.E. Ortega, ${ }^{1,3,2}$ and D. G. de Oteyza ${ }^{3, *}$ \\ ${ }^{1}$ Donostia International Physics Center, Paseo Manuel Lardizabal 4, E-20018 San Sebastián, Spain \\ ${ }^{2}$ Departamento Física Aplicada I, Universidad del País Vasco, 20018 San Sebastián, Spain \\ ${ }^{3}$ Centro de Física de Materiales (CSIC/UPV-EHU)-Materials Physics Center (MPC), \\ Paseo Manuel Lardizabal 5, 20018 San Sebastián, Spain \\ ${ }^{4}$ European Synchrotron Radiation Facility, BP 220, 6 rue Jules Horowitz, 38043 Grenoble, France
}

(Received 21 November 2013; revised manuscript received 14 January 2014; published 18 March 2014; corrected 31 March 2014)

Codeposition of two molecular species [copper phtalocyanine ( $\mathrm{CuPc}$, donor) and perfluoropentacene (PFP, acceptor)] on noble metal (111) surfaces leads to the self-assembly of an ordered mixed layer with a maximized donor-acceptor contact area. The main driving force behind this arrangement is assumed to be the intermolecular $\mathrm{C}-\mathrm{H} \cdots \mathrm{F}$ hydrogen-bond interactions. Such interactions would be maximized for a coplanar molecular arrangement. However, precise measurement of molecule-substrate distances in the molecular mixture reveals significantly larger adsorption heights for PFP than for CuPc. Most surprisingly, instead of leveling to increase hydrogen-bond interactions, the height difference is enhanced in the blends as compared to the heights found in single-component CuPc and PFP layers. The increased height of PFP in mixed layers points to an overall reduced interaction with the underlying substrate, and its influence on electronic properties like the interface dipole is investigated through work function measurements.

Charge carrier injection in organic optoelectronic devices depends on the electronic properties of metal-organic interfaces, which are, therefore, of utmost importance for device efficiency. Such properties are strongly affected by the detailed interfacial structure defined by the molecular orientation with respect to the substrate, the lateral distribution, and the molecule-substrate distance. The latter, in particular, plays a central role in the interfacial energy level alignment. It affects strong chemical interactions and their associated electronic changes [1], band gap renormalizations through image potential effects [2], as well as a number of other processes determining potential changes at nonchemisorptive interfaces. These are described, for example, in the electronic interface model put forward by Vázquez and co-workers [2-4], which includes the effects of permanent molecular dipoles, the "pillow effect," and interfacial charge transfer. All of them sum up to render the so-called interface dipole, and, in particular, the two latter strongly depend on the molecule-substrate distance [2-4]. The most precise technique to determine this distance experimentally is normal incidence $\mathrm{x}$-ray standing waves (XSW) [5-7], by means of which the interfaces of a number of semiconducting molecules with various surfaces have been studied [1,8-16].

Going beyond single-component layers, here we report on the use of XSW to determine the molecule-substrate distances in donor-acceptor molecular blends, which are not only highly relevant for many organic devices but also have interfacial properties often differing from those of the corresponding single-component layers [17-19]. We provide a complete structural characterization of a stoichiometric 1:1 monolayer blend of copper phtalocyanine (CuPc, donor) and perfluoropentacene (PFP, acceptor) on the $\mathrm{Ag}(111)$ and $\mathrm{Cu}(111)$ substrates. The lateral order of the 2D blends and the molecule-substrate distances have been characterized using scanning tunneling microscopy (STM) and XSW, respectively. The former evidences highly crystalline mixed monolayers, while the latter reveals how the molecules in the blends significantly change their molecule-substrate distances with respect to those in singlecomponent layers. Finally, we analyze the impact of those changes on the interface electronic properties by means of photoemission work function measurements.

Constant current STM images show that depositing PFP, $\mathrm{CuPc}$, or molecular blends on $\mathrm{Ag}(111)$ and $\mathrm{Cu}(111)$ leads to the self-assembly of ordered molecular layers in which molecules adopt a flat-lying configuration. The structure of PFP or $\mathrm{CuPc}$ monolayers on $\mathrm{Ag}(111)$ and $\mathrm{Cu}(111)$ have already been reported in the literature [12,20-24]. We, therefore, place our focus on the mixed layers. When codeposited (or deposited sequentially one after the other, which renders the same results) in an approximately $1: 1$ ratio, the two molecules form an ordered structure similar to that found for closely related systems $[17,25,26]$, in which molecules of one type surround themselves by the other type maximizing donor-acceptor contact and $\mathrm{C}-\mathrm{H} \cdots \mathrm{F}$ intermolecular hydrogen bonds (Fig. 1). The experimental unit cell parameters are summarized in 
(a)
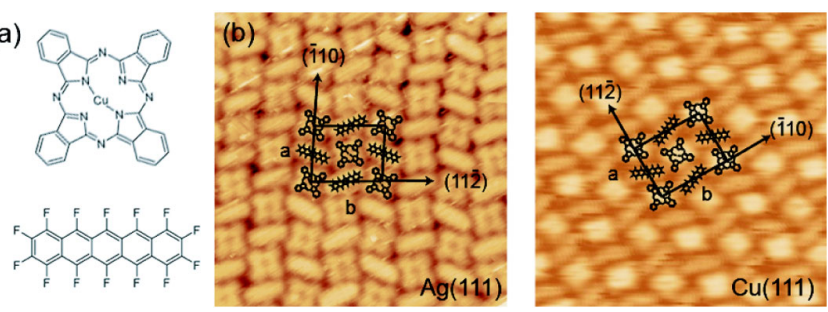

FIG. 1 (color online). (a) Chemical structure of CuPc (above) and PFP (below). (b) and (c) $11.5 \mathrm{~nm} \times 11.5 \mathrm{~nm}$ images of the 1:1 PFP $+\mathrm{CuPc}$ blend on $\mathrm{Ag}(111)$ and $\mathrm{Cu}(111)$, respectively. The unit cell, substrate directions, and molecular schemes are overlaid on the images.

Table I. These, in combination with the discrete azimuthal domain orientations measured, allow us to put forth rectangular commensurate structures as tentative epitaxial models (see Table I).

Normal incidence $\mathrm{x}$-ray standing wave measurements of the donor-acceptor mixtures have been performed at the beamline ID32 of the ESRF probing the $\mathrm{C}, \mathrm{N}$, and $\mathrm{F}$ atoms and are summarized in Fig. 2. Because of the molecular compositions, the photoelectron signal of the $\mathrm{N}$ and $\mathrm{F}$ atoms can be unambiguously ascribed to $\mathrm{CuPc}$ and PFP, respectively. However, a more complex scenario is found for $\mathrm{C}$, which is contained in both molecules. We disentangle the signal from each molecule in the mixture's XSW photoemission spectra based on previous high-resolution XPS studies [Fig. 2(a)] performed at the beamline ALOISA of the synchrotron Elettra [17]. Three separate peaks are resolved [Fig. 2(a)]. From highest to lowest binding energies, these correspond to PFP's $\mathrm{C}_{\mathrm{F}}$ component (carbon atoms bound to fluorine), PFP's $\mathrm{C}_{\mathrm{C}}$ component (carbon atoms bound solely to carbon) convolved with CuPc's $\mathrm{C}_{\mathrm{N}}$ component (carbon atoms bound to nitrogen), and CuPc's remaining components, $\mathrm{C}_{\mathrm{C}}$ and $\mathrm{C}_{\mathrm{H}}$ (carbon atoms bound solely to carbon or also hydrogen). In our XSW analysis, we only consider $\mathrm{C}_{\mathrm{F}}$ in $\mathrm{PFP}$ and $\mathrm{C}_{\mathrm{C} / \mathrm{H}}$ in $\mathrm{CuPc}$, which can each be fitted by a single peak and are expected to give the most accurate results; the second peak in which the components of both molecules overlap is disregarded.

Figure 2(b) shows examples of a reflectivity curve (red) and of the photoelectron yield curves (green) for CuPc's $\mathrm{C}_{\mathrm{C} / \mathrm{H}}$ and $\mathrm{N}$, as well as PFP's $\mathrm{C}_{\mathrm{F}}$ and $\mathrm{F}$ obtained for each

TABLE I. Summary of STM results including unit cell parameters and epitaxy matrices (E.M.) with base vectors (110) and (112) [27].

\begin{tabular}{lcccc}
\hline \hline & \multicolumn{2}{c}{$\mathrm{Ag}(111)$} & \multicolumn{2}{c}{$\mathrm{Cu}(111)$} \\
& Experiment & Model & Experiment & Model \\
\hline$a$ & $22 \pm 2 \AA$ & $23.1 \AA$ & $21 \pm 1 \AA$ & $22.2 \AA$ \\
$b$ & $29 \pm 1 \AA$ & $30.0 \AA$ & $27 \pm 2 \AA$ & $28.2 \AA$ \\
$\alpha$ & $89^{\circ} \pm 6^{\circ}$ & $90^{\circ}$ & $89^{\circ} \pm 5^{\circ}$ & $90^{\circ}$ \\
E.M. & \multicolumn{2}{c}{$(8,0 / 0,6)$} & \multicolumn{2}{c}{$(0,5 / 11,0)$} \\
\hline \hline
\end{tabular}
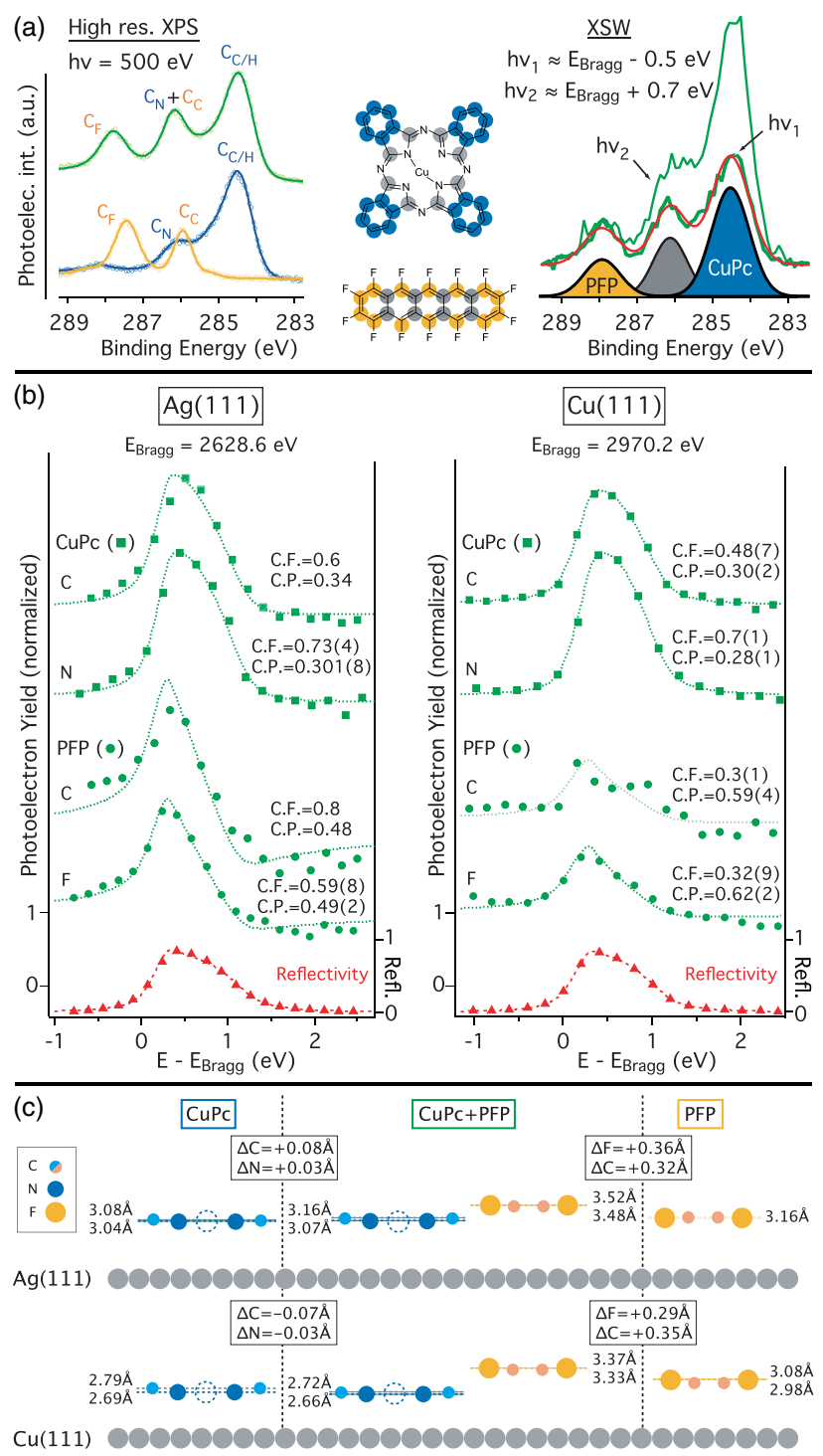

FIG. 2 (color online). (a) Left: High-resolution $\mathrm{C}_{1 \mathrm{~s}}$ photoemission intensity of single-component and mixed layers on $\operatorname{Ag}(111)(\mathrm{CuPc}=$ blue, $\mathrm{PFP}=$ yellow, $\operatorname{mix}=$ green $)$. Right: Also on $\operatorname{Ag}(111), C_{1 s}$ photoemission intensity of the mix at photon energies corresponding to the minimum $\left(h \nu_{1}\right)$ and close to the maximum $\left(h \nu_{2}\right)$ of the reflectivity curve. The fitted curve (red) is made up of three Gaussians, each corresponding to the different chemical environments of the carbon atoms in the molecules: PFP's $\mathrm{C}_{\mathrm{F}}$ component (yellow filling), $\mathrm{CuPc} \mathrm{C}_{\mathrm{H}}$ and $\mathrm{C}_{\mathrm{C}}$ components (blue filling), and a convolution of PFP's $\mathrm{C}_{\mathrm{C}}$ and CuPc's $\mathrm{C}_{\mathrm{N}}$ (grey filling). The associated atoms are marked correspondingly in the molecular diagrams in the center. (b) Reflectivity curve (red triangles) and $\mathrm{C}_{1 \mathrm{~s}}, \mathrm{~N}_{1 \mathrm{~s}}$, and $\mathrm{F}_{1 \mathrm{~s}}$ photoelectron yield curves (green curves; those of $\mathrm{C}$ and $\mathrm{N}$ are offset for clarity) for the molecular mixture on $\operatorname{Ag}(111)$ (left) and $\mathrm{Cu}(111)$ (right). (c) Molecular adsorption heights of $\mathrm{CuPc}$ monolayers (left) [12,13], PFP monolayers (right) $[10,11]$, and the PFP + CuPc mix (center) on the $\mathrm{Ag}$ (111) (top) and $\mathrm{Cu}(111)$ (bottom) surfaces, including height changes in the mixed layers referred to the single-component monolayers. Error in $d_{H}$ is about $0.05 \AA$ [27]. Distance to the substrate is not to scale. 
substrate [28]. The lower quality of the data corresponding to PFP's carbon [yellow Gaussian in Fig. 2(a)] can be explained by a worse signal-to-noise ratio in the core-level spectra. From the fitting of the yield curves, we obtain the coherent fraction (C.F.) related to the degree of vertical order and coherent position (C.P.) related to the adsorbatesubstrate distance $d_{H}$ [5-7].

The coherent fractions obtained are, within the error margins, similar to those previously reported for the associated single-component layers [10-13]. The only exception is PFP on $\operatorname{Ag}(111)$, for which the C.F. is greatly enhanced in the mixed layer, related to the change from an incommensurate PFP monolayer displaying a moiré pattern (at room temperature) [20], to a commensurate structure. The fact that the PFP's C.F. in the blends on $\operatorname{Ag}(111)$ reaches values considerably higher than on $\mathrm{Cu}(111)$ might, in turn, be related to a smaller buckling resulting from a weaker interaction with $\operatorname{Ag}(111)$ [15]. The obtained $d_{H}$ values [27] and a schematic representation of the molecular heights in the mix are shown in Fig. 2(c). The heights previously reported for single-component $\mathrm{CuPc}$ and PFP layers [10-13] are also included for comparison.

The molecule-substrate distance generally mirrors the interaction strength between the molecule and the underlying surface. This has been reported, for instance, for $\mathrm{CuPc}$, PTCDA, or DIP, all of which show a clear trend of decreasing height on increasingly interactive substrates when going from $\mathrm{Au}(111)$ through $\mathrm{Ag}(111)$ to $\mathrm{Cu}(111)$ [12-15]. In line with those observations, all our measured heights show consistently lower values on $\mathrm{Cu}(111)$ than on $\operatorname{Ag}(111)$. However, disregarding this difference, the results on $\mathrm{Ag}(111)$ and $\mathrm{Cu}(111)$ are qualitatively similar: the most pronounced changes comparing single-component [10-13] and mixed layers are found in the acceptor molecule PFP. Our analysis reveals a substantial height change in both the $\mathrm{C}_{\mathrm{F}}$ and $\mathrm{F}$ atoms, suggesting that the entire molecule is raised $\sim 0.3 \AA$ from the surface in the mixture. In contrast, neither the height of $\mathrm{CuPc}$ as a whole nor its configuration (with $\mathrm{N}$ atoms at slightly lower height than the $\mathrm{C}$ atoms) show substantial changes.

In single-component layers, $\mathrm{CuPc}$ lies closer to the substrate than PFP, both on $\mathrm{Ag}(111)$ and $\mathrm{Cu}(111)$ [10-13]. Upon blend formation, the raising up of PFP further increases the height difference between donors and acceptors [Fig. 2(c)]. This is contrary to expectations, since hydrogen bonding strength is known to increase with bond linearity and with shorter bond lengths $[29,30]$. The intermolecular C-H $\cdots \mathrm{F}$ bonds assumed to drive the self-assembly would, therefore, be strongest in a coplanar arrangement and tend to level the molecular heights in the blends. Consequently, other driving forces must be behind these surprising changes. Substrate-mediated effects seem most intuitive and may arise from changes in the interface electronics related to the new supramolecular environment in the blends $[17,18]$, which, in turn, modify the molecule-substrate interactions and the associated adsorption distances.

On the whole, the increased molecule-substrate distance of PFP and unchanged distance of $\mathrm{CuPc}$ suggest an overall reduced interaction of the mixed molecular layer with the underlying substrate. Reduced molecule-substrate interactions as a result of enhanced intermolecular interactions in molecular mixtures have been reported before [31,32] and show an analogy to coordination chemistry or Pauling's rules, where increased coordination numbers (interactions with substrate and surrounding molecules vs only with the substrate) weaken the strength of each single bond [33]. However, this is the first quantitative report on the associated changes in the molecule-substrate distances of molecular blends, which are of great importance for the understanding of the interfacial electronic properties $[2-4,17]$.

The changes in adsorption height found in the mixed layer are expected to lead to variations in the interface dipole. These variations are measurable as changes in the system's work function and are typically obtained from the secondary electron cutoff in ultraviolet photoemission spectra (UPS) [34,35]. The work function dependence on coverage was first determined for each molecule on $\mathrm{Ag}(111)$ and $\mathrm{Cu}(111)$ by first measuring that of the clean substrate as reference and performing further measurements after each subsequent step in the evaporation. The results are shown in Fig. 3(a). As expected in nonpolar molecules [34,35], in all cases, the work function is found to change steadily up to certain "saturation" coverage associated with a completely covered surface [monolayer (ML) coverage], after which it remains practically constant. The work function shift for $1 \mathrm{ML}$ on $\mathrm{Ag}(111)$ and $\mathrm{Cu}(111)$ was found to be -0.41 and $-0.33 \mathrm{eV}$ for PFP and -0.44 and $-0.72 \mathrm{eV}$ for CuPc. Previously reported values, available to the best of our knowledge only for the PFP-based interfaces, are in good agreement with our results $[10,11]$.

We now turn our attention to the work function of the mixed layers. Figure 3(b) shows the vacuum level shifts associated with the deposition of the $\mathrm{CuPc}+\mathrm{PFP}$ molecular mixture and of just PFP. The same quantity of PFP (namely, approximately the one needed to form the stoichiometric 1:1 molecular blend) is found to shift the vacuum level by different amounts when deposited on the submonolayer $\mathrm{CuPc} /$ metal (rendering a mixed layer) and on the clean metal [36]. On $\mathrm{Cu}(111)$, 0.42 ML of PFP was deposited onto $0.48 \mathrm{ML}$ $\mathrm{CuPc} / \mathrm{Cu}(111)$ producing a shift of $\Delta \Phi_{\text {mix }}(\mathrm{Cu})=$ $-0.10 \mathrm{eV}$ in the work function. From the known coverage dependence of the work function of the PFP/Cu(111) system, we find that $\Delta \Phi_{\text {mix }}(\mathrm{Cu})$ is an $\sim 40 \%$ reduction of the expected value for a direct 0.42 ML PFP deposition on $\mathrm{Cu}(111) \Delta \Phi_{\text {pure }}(\mathrm{Cu})=-0.17 \mathrm{eV}$. The scenario on $\mathrm{Ag}$ (111) is very similar: 0.32 ML of PFP deposited onto 

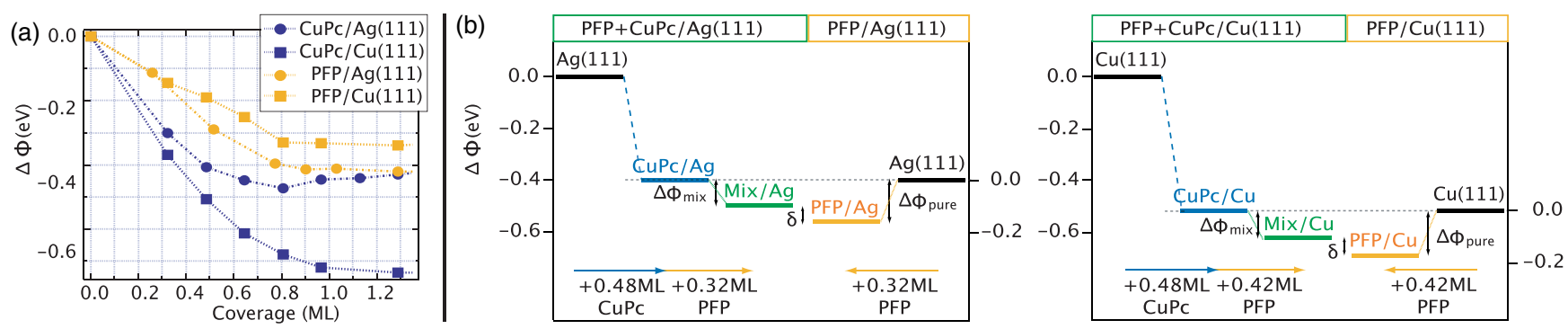

FIG. 3 (color online). (a) Work function changes associated with CuPc (blue) and PFP (yellow) on $\mathrm{Ag}(111)$ (round markers) and $\mathrm{Cu}(111)$ (square markers) substrates as a function of coverage. (b) The work function change $(\Delta \Phi)$ caused by deposition of a given amount of PFP is reduced by an amount $\delta$ when PFP is deposited upon a precovered submonolayer CuPc/metal system $\left(\Delta \Phi_{\text {mix }}\right)$, as compared to deposition on the clean metal $\left(\Delta \Phi_{\text {pure }}\right)$. The effect is observed on both $\operatorname{Ag}(111)$ (left) and $\mathrm{Cu}(111)$ (right). The blue bar $(\mathrm{CuPc} / \mathrm{metal})$ and the black bar (clean metal) have been aligned in order to more easily compare $\Delta \Phi_{\text {mix }}$ and $\Delta \Phi_{\text {pure }}$.

0.48 ML CuPc/ $\operatorname{Ag}(111)$ causes the work function to shift downwards by $\Delta \Phi_{\text {mix }}(\mathrm{Ag})=-0.10 \mathrm{eV}$ compared to an expected value of $\Delta \Phi_{\text {pure }}(\mathrm{Ag})=-0.16 \mathrm{eV}$. Again, a reduction of about $40 \%$ is found [27].

Reciprocal depolarization of nearby dipoles [35], i.e., depolarization of CuPc by mixing with PFP, could partially explain the reduced work function variation observed when PFP is added to the $\mathrm{CuPc} / \mathrm{Ag}(111)$ interface. However, as demonstrated by the XSW measurements, geometry changes do not affect $\mathrm{CuPc}$ molecules, which strongly suggests that the $\mathrm{CuPc}$ dipole does not vary upon mixing. We may, thus, assume that only the PFP dipole changes when going from pure layers to the mixture.

The change in the effective PFP dipole may be obtained from the experimentally determined work function changes $\Delta \Phi_{\text {pure }}$ and $\Delta \Phi_{\text {mix }}$ by using the Helmholtz equation

$$
\Delta \Phi=\frac{e P n}{\epsilon \epsilon_{0}},
$$

[10,35] where $P$ is the effective dipole moment per molecule, $n$ is the areal density of dipoles [37], $e$ is the elementary charge, $\epsilon$ is the relative dielectric constant $(\epsilon \sim 1[10,35])$, and $\epsilon_{0}$ is the vacuum permittivity. We estimate that upon mixing, the effective dipole moment associated with PFP changes from $P_{\text {pure }}=1.75 \mathrm{D}$ to $P_{\text {mix }}=1.10 \mathrm{D}$ on $\operatorname{Ag}(111)$ and from $P_{\text {pure }}=1.52 \mathrm{D}$ to $P_{\text {mix }}=0.90 \mathrm{D}$ on $\mathrm{Cu}(111)$.

The most important effects that contribute to the interface dipole are (i) the Pauli repulsion between the molecule's orbitals and the metal's electrons decaying into vacuum, (ii) charge transfer between molecule and substrate, and (iii) the molecules' intrinsic electric dipole moment [38]. We discard option (ii) as a possible explanation for the observed changes, since charge transfer values calculated on $\operatorname{Ag}(111)$ [39] indicate that this effect results in a net dipole change in the opposite direction. Option (iii) is, likewise, ruled out as a main contributor to the reduction in $P$, since changes in the intrinsic dipolar moment in the mix due to modified molecular distortions lead to net dipole changes in opposite directions on $\mathrm{Ag}(111)$ and on $\mathrm{Cu}(111)$ [on $\mathrm{Ag}(111)$, when going from a single-component layer to a mixed layer, the net change in intramolecular dipole points into the surface, whereas on $\mathrm{Cu}(111)$, it points away from it]. We argue that while all contributions are present, only the Pauli repulsion (i) can explain the common trend observed on $\operatorname{Ag}(111)$ and $\mathrm{Cu}(111)$ and must, therefore, be the effect contributing the most to the experimentally observed changes in $P$ : the increased molecule-substrate distance of PFP found in the mixture translates into a reduced Pauli repulsion, thereby decreasing the effective interface dipole.

In conclusion, we have characterized the lateral and vertical structures of $\mathrm{CuPc}$ and PFP molecular mixtures in a 1:1 ratio on $\mathrm{Ag}(111)$ and $\mathrm{Cu}(111)$ substrates. Contrary to what might be expected in a molecular blend stabilized by hydrogen bonding, XSW measurements revealed that $\mathrm{CuPc}$ and PFP lie at considerably different heights. Most strikingly, that difference is enhanced in the mixed layers as compared to the respective heights in single-component layers. While $\mathrm{CuPc}$ remained virtually at the same height upon mixing, PFP was found to raise up substantially $(\sim 0.3 \AA)$ on both the $\mathrm{Ag}(111)$ and $\mathrm{Cu}(111)$ substrates. Such a change in the adsorption height of PFP is expected to affect interface phenomena. Our photoemission measurements show this effect is, indeed, measurable, as we find that the work function shift caused by deposition of PFP onto $\mathrm{CuPc} /$ metal to form the molecular blend is smaller than the shift found for the deposition of the same amount of PFP onto the bare metal substrate. We hereby provide a direct measure of the effect of a molecule's adsorption height on vacuum level shifts and, in turn, interfacial energy level alignment.

This work was supported by the Spanish Grants No. MAT2010-21156-C03-01 and-C03-03, as well as No. PIB2010US-00652, and by the Basque Government (Grant No. IT-621-13). D. G. O. acknowledges support from the European Union under Grant No. FP7PEOPLE-2010-IOF-271909. We acknowledge funding from the European Community's Seventh Framework Programme (FP7/2007-2013) under Grant No. 226716. 
We thank the European Synchrotron Radiation Facility (ESRF) for provision of synchrotron radiation facilities, J. Zegenhagen, H. Isern, and L. André for support of the XSW beamline, and J. Roy for his help with the pyXSW data analysis software. E. G. thanks J. G. Goiri for technical assistance.

*Corresponding author.d_g_oteyza@ehu.es

${ }^{\dagger}$ Present address: CEA-LETI, MINATEC Campus, 17 rue des Martyrs, 38054 Grenoble, France.

[1] G. Heimel, S. Duhm, I. Salzmann, A. Gerlach, A. Strozecka, J. Niederhausen, C. Bürker, T. Hosokai, I. FernandezTorrente, G. Schulze et al., Nat. Chem. 5, 187 (2013).

[2] F. Flores, J. Ortega, and H. Vazquez, Phys. Chem. Chem. Phys. 11, 8658 (2009).

[3] H. Vázquez, Y. J. Dappe, J. Ortega, and F. Flores, J. Chem. Phys. 126, 144703 (2007).

[4] M. G. Betti, A. Kanjilal, C. Mariani, H. Vázquez, Y. J. Dappe, J. Ortega, and F. Flores, Phys. Rev. Lett. 100, 027601 (2008).

[5] D. P. Woodruff, Prog. Surf. Sci. 57, 1 (1998).

[6] J. Zegenhagen, Surf. Sci. Rep. 18, 202 (1993).

[7] A. Gerlach, C. Bürker, T. Hosokai, and F. Schreiber, The Molecule-Metal Interface (Wiley-VCH, Singapore, 2013), pp. 153-172.

[8] S. Henze, O. Bauer, T.-L. Lee, M. Sokolowski, and F. Tautz, Surf. Sci. 601, 1566 (2007).

[9] A. Gerlach, F. Schreiber, S. Sellner, H. Dosch, I. A. Vartanyants, B. C. C. Cowie, T.-L. Lee, and J. Zegenhagen, Phys. Rev. B 71, 205425 (2005).

[10] N. Koch, A. Gerlach, S. Duhm, H. Glowatzki, G. Heimel, A. Vollmer, Y. Sakamoto, T. Suzuki, J. Zegenhagen, J. P. Rabe et al., J. Am. Chem. Soc. 130, 7300 (2008).

[11] S. Duhm, S. Hosoumi, I. Salzmann, A. Gerlach, M. Oehzelt, B. Wedl, T.-L. Lee, F. Schreiber, N. Koch, N. Ueno et al., Phys. Rev. B 81, 045418 (2010).

[12] I. Kröger, B. Stadtmüller, C. Kleimann, P. Rajput, and C. Kumpf, Phys. Rev. B 83, 195414 (2011).

[13] I. Kröger, B. Stadtmüller, C. Stadler, J. Ziroff, M. Kochler, A. Stahl, F. Pollinger, T.-L. Lee, J. Zegenhagen, F. Reinert et al., New J. Phys. 12, 083038 (2010).

[14] C. Bürker, N. Ferri, A. Tkatchenko, A. Gerlach, J. Niederhausen, T. Hosokai, S. Duhm, J. Zegenhagen, N. Koch, and F. Schreiber, Phys. Rev. B 87, 165443 (2013).

[15] S. Duhm, A. Gerlach, I. Salzmann, B. Bröker, R. Johnson, F. Schreiber, and N. Koch, Org. Electron. 9, 111 (2008).

[16] C. Stadler, S. Hansen, I. Kröger, C. Kumpf, and E. Umbach, Nat. Phys. 5, 153 (2009).

[17] A. El-Sayed, P. Borghetti, E. Goiri, C. Rogero, L. Floreano, G. Lovat, D. J. Mowbray, J. L. Cabellos, Y. Wakayama, A. Rubio et al., ACS Nano 7, 6914 (2013).

[18] D. G. de Oteyza, J. M. García-Lastra, B. P. D. M. Corso, L. Floreano, A. Morgante, Y. Wakayama, A. Rubio, and J. E. Ortega, Adv. Funct. Mater. 19, 3567 (2009).

[19] J.-Q. Zhong, X. Qin, J.-L. Zhang, S. Kera, N. Ueno, A. T. S. Wee, J. Yang, and W. Chen, ACS Nano 8, 1699 (2014).
[20] E. Goiri, J. M. García-Lastra, M. Corso, Z. M. Adb El-Fattah, J. E. Ortega, and D. G. de Oteyza, J. Phys. Chem. Lett. 3, 848 (2012).

[21] M. Marks, C. Schmidt, C. H. Schwalb, T. Breuer, G. Witte, and U. Höfer, J. Phys. Chem. C 116, 1904 (2012).

[22] H. Glowatzki, G. Heimel, A. Vollmer, S. L. Wong, H. Huang, W. Chen, A. T. S. Wee, J. P. Rabe, and N. Koch, J. Phys. Chem. C 116, 7726 (2012).

[23] H. Karacuban, M. Lange, J. Schaffert, O. Weingart, T. Wagner, and R. Müller, Surf. Sci. 603, L39 (2009).

[24] J. C. Buchholz and G. A. Somorjai, J. Chem. Phys. 66, 573 (1977).

[25] Y. Wakayama, D. G. de Oteyza, J. M. García-Lastra, and D. J. Mowbray, ACS Nano 5, 581 (2011).

[26] E. Barrena, D. G. de Oteyza, H. Dosch, and Y. Wakayama, ChemPhysChem 8, 1915 (2007).

[27] See Supplemental Material at http://link.aps.org/ supplemental/10.1103/PhysRevLett.112.117602 for experimental details, discussion of errors in STM, XSW, and UPS, and an assessment of the effects of beam damage in XSW.

[28] As each atomic species was measured at more than one spot on the sample, the reflectivity curves shown in Fig. 2 are two of several and correspond to the $F_{1 s}$ yield curves shown in the figure.

[29] T. Steiner, Angew. Chem., Int. Ed. Engl. 41, 48 (2002).

[30] G. R. Desiraju, Acc. Chem. Res. 35, 565 (2002).

[31] K. J. Franke, G. Schulze, N. Henningsen, I. FernándezTorrente, J. I. Pascual, S. Zarwell, K. Rück-Braun, M. Cobian, and N. Lorente, Phys. Rev. Lett. 100, 036807 (2008).

[32] D. G. de Oteyza, I. Silanes, M. Ruiz-Osés, E. Barrena, B. P. Doyle, A. Arnau, H. Dosch, Y. Wakayama, and J. E. Ortega, Adv. Funct. Mater. 19, 259 (2009).

[33] X. Guo, L.-M. Wang, B. Xu, Z. Liu, D. Yu, J. He, H.-T. Wang, and Y. Tian, J. Phys. Condens. Matter 21, 485405 (2009).

[34] A. Kahn, N. Koch, and W. Gao, J. Polym. Sci. Pol. Phys. 41, 2529 (2003).

[35] H. Fukagawa, S. Hosoumi, H. Yamane, S. Kera, and N. Ueno, Phys. Rev. B 83, 085304 (2011).

[36] Because of the different molecular sizes, less PFP coverage than $\mathrm{CuPc}$ is required for a $1: 1$ ratio. Nominal deposition of $0.32 \mathrm{ML}$ PFP on $0.48 \mathrm{ML} \mathrm{CuPc} / \mathrm{Ag}$ gave, as obtained from a statistical analysis of 14 STM images, $72 \%$ of the surface covered with a 1:1 mixture, $2 \%$ with patches of ordered PFP, and the remaining 26\% empty, in good agreement with the nominal deposition thicknesses. In turn, 0.42 ML PFP on $0.48 \mathrm{ML} \mathrm{CuPc} / \mathrm{Cu}$ implies a minor excess of PFP with respect to a stoichiometric $1: 1$ mixture, rendering a small amount $(\sim 10 \%)$ of coexisting pure PFP patches.

[37] $n=n_{\mathrm{ML}} \Theta$, where $\Theta$ is the coverage of PFP and $n_{\mathrm{ML}}$ the dipole density for full monolayer coverage, which is $1 / 132 \AA^{2}$ for $\mathrm{PFP} / \mathrm{Ag}(111)$, and $1 / 142 \AA^{2}$ for PFP/Cu(111), as determined from STM measurements of the respective monolayers.

[38] H. Ishii, K. Sugiyama, E. Ito, and K. Seki, Adv. Mater. 11, 605 (1999).

[39] J. L. Cabellos, D. J. Mowbray, E. Goiri, A. El-Sayed, L. Floreano, D. G. de Oteyza, C. Rogero, J. E. Ortega, and A. Rubio, J. Phys. Chem. C 116, 17991 (2012). 